\title{
The Epidemiologic Charateristics, Healthcare Associated and Household Transmission Dynamics of EVD Outbreak in a South-Southern City of Nigeria
}

\author{
Olawunmi O. Adeoye ${ }^{\star 1}$, Endie Waziri', Uchenna Anebonam¹, Ifeoma Nwaduito ${ }^{2}$, Pauline \\ Green $^{2}$, William Komakech ${ }^{3}$, Nnanna Onyekwere ${ }^{2}$, Gabriele Poggensee ${ }^{1}$ and Patrick M. \\ Nguku $^{1}$
}

${ }^{1}$ Nigeria Field Epidemiology and Laboratory Training Programme, Asokoro, Nigeria; ${ }^{2}$ Rivers State Ministry of Health, PortHarcourt, Nigeria; ${ }^{3}$ World Health Organisation, South South Zone, Nigeria

\section{Objective}

This study describes the epidemiological characteristics and the transmission dynamics of the EVD outbreak in a South-Southern city of Nigeria

\section{Introduction}

Ebola virus disease (EVD) is a severe illness that spread in the human population through human-to-human transmission. In the past, EVD outbreaks occurred in the rural communities of Africa, near tropical rainforests, but the most recent outbreak in West Africa has also involved major urban areas and big cities, with air travel playing an important role in its spread.On July 23, 2014, the EVD outbreak was declared in Nigeria following the confirmation of EVD in a traveller, who arrived acutely ill at the international airport in Lagos, South Western Nigeria from Liberia .The outbreak subsequently filtered to a South Southern Nigeria city, by a symptomatic contact who escaped surveillance in Lagos and flew to the South Southern city.

\section{Methods}

A detailed case investigation was initiated on the $27^{\text {th }}$, August, 2014 to confirm the alert of a possible outbreak in the South Southern city of the death of a physician from illness with symptoms compatible of EVD. The possible source of exposure in the deceased physician was explored by tracing his contacts retrospectively. Stored sample of the physician's blood taken while he was ill was also sent for laboratory analysis. Standardised case and contact definitions were adopted. Contact identification, listing and follow up was done till the $21^{\text {st }}$ after the last possible exposure with immediate isolation of cases. Analysis for confirmation of EVD was also done using RT-PCR.

\section{Results}

The stored sample of the physician's blood was positive for EVD on RT-PCR. The retrospective tracing of the deceased physician's contact revealed he managed a patient, a contact of a confirmed case in another city of Nigeria, who escaped surveillance, and flew into the South-Southern city discreetly by plane while already very ill with symptoms of Ebola in the first week of August. This patient was said to have been managed in a hotel room by the physician for 5 days. Three secondary cases arose from this index case (the deceased physician). The deceased physician transmitted the virus to a patient he shared a hospital room with for 2 nights during the course of his illness. None of the health care workers that took care of him in the hospital was infected. He also generated 2 other cases within his household (his wife and sister) who were both involved in caring for him at home while he was ill, giving a total of 4 cases with 527 contacts listed in all (attack rate $=7.6 / 1000)$. There were two deaths (case fatality ratio $=50 \%$ ). The mean incubation period was 13 days \pm 4 days and average serial interval was 18 days \pm 2 days

\section{Conclusions}

Infection Prevention and Control measures in most health care facilities in Nigeria are focused on protecting health care workers from infected patients with little consideration on preventing cross infections among patients. Active surveillance and screening of passengers should not only be limited to international ports of entry but should be promptly and strictly enforced at domestic airports and inter-state borders as soon as an outbreak is declared to limit the spread of the outbreak locally.

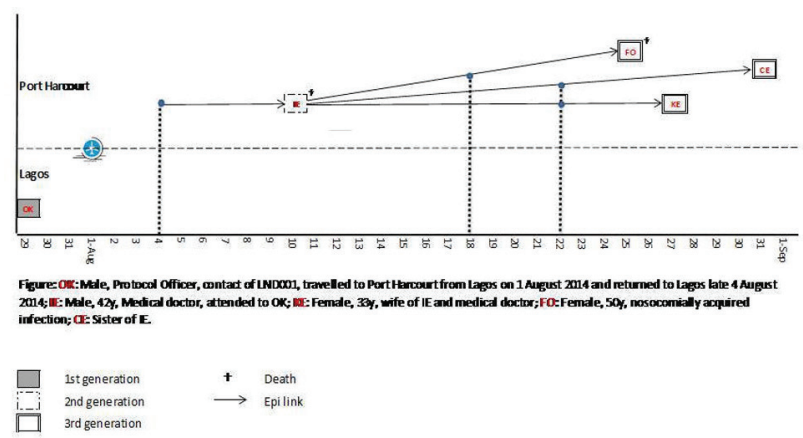

THE EVD TRANSMISSION CHAIN IN PORTHARCOURT, SOUTH SOUTHERN NIGERIA.

\section{Keywords}

Epidemiologic characteristics; Transmission dynamics; EVD outbreak; Nigeria

\section{*Olawunmi O. Adeoye}

E-mail: wunmiolat@yahoo.com 